\title{
Veneers - Indirectly Fabricated Dental Porcelain for Aesthetic
}

\author{
Attur Kailash ${ }^{1}$, Nikibahen Modi ${ }^{*}$, Nikunj Patel ${ }^{3}$, Shruti Patel ${ }^{4}$ \\ ${ }^{1}$ Professor and Head' Department of Conservative Dentistry and Endodontics, Narsinhbhai Patel Dental College \\ and Hospital, Visnagar, India \\ ${ }^{2}$ Post Graduate Student, Department of Conservative Dentistry and Endodontics, Narsinhbhai Patel Dental \\ College and Hospital, Visnagar, India \\ ${ }^{3}$ Reader, Department of Conservative Dentistry and Endodontics, Narsinhbhai Patel Dental College and \\ Hospital, Visnagar, India \\ ${ }^{4}$ Ex-Post Graduate Student, Department of Conservative Dentistry and Endodontics, Narsinhbhai Patel Dental \\ College and Hospital, Visnagar, India \\ *Corresponding Author: Nikibahen Modi, Department of Conservative Dentistry and Endodontics, \\ Narsinhbhai Patel Dental College and Hospital, Visnagar, India; Email: nikimodi34@yahoo.in
}

Received Date: 25-10-2021; Accepted Date: 16-10-2021; Published Date: 23-11-2021

Copyright $^{\circledR} 2021$ by Modi N, et al. All rights reserved. This is an open access article distributed under the terms of the Creative Commons Attribution License, which permits unrestricted use, distribution and reproduction in any medium, provided the original author and source are credited.

\begin{abstract}
Indirect veneers are the thin films that stick directly to the facial surfaces of the teeth to develop its aesthetics. Indirect veneer is simply enveloping conservative and tremendous aesthetic restoration which has developed over the more than a few years. This case report shows teeth having fluorosis in anterior maxillary arch and are treated by best conservative aesthetic solution using indirect technique. At the present time, it is very essential to establish beautiful aesthetic smile without affecting or loosing much sound tooth structure and indirect veneers are the best long term solution for this. In this case report all clinical steps with laboratory part has been covered.
\end{abstract}

\section{Keywords}

Aesthetic Rehabilitation; Indirect Veneer; Dental Fluorosis 


\section{Introduction}

Indirect porcelain laminate veneers are one of the most esthetic and conventional restoration. In indirect technique the teeth affected with fluorosis are bonded with a thin ceramic restoration of porcelain veneers and restores the facial surface of teeth which requires esthetic correction [1]. Innumerable previous studies have proved that bonded indirectly fabricated dental poecelain veneer gave best outcome over a time of 10 years [1]. In 1938 porcelain veneers were introduced by Charles Pincus to give temporary aesthetic to patients. Overlong porcelain veneers have brilliant aesthetics. Plaque observance is not as much of because of the tissue compatibility and impenetrable nature of the porcelain, with no damaging effect on gingiva [2]. For obtaining best result proper analysis of case should be done prior to start tooth preparation. By this analysis it can be make sure that the teeth being prepared will require very less tooth preparation [2]. Outstanding perfection properties with mechanical strength have been provided by porcelain veneers with conservation of tooth structure. Thus teeth which requires esthetic improvement like fluorosis, stains of tetracycline, midline diastemas, spacing in anterior teeth can be corrected with this technique [3]. The goal of esthetic dentistry should be "bright, beautiful, but believable" [4]. These veneers have a number of advantages such as fracture resistance, prevent attrition of teeth and discolorations compared to direct restorations technique. There are several hurdles for this technique like longer time appointment, high costing and also requires adhesive system for cementation [5]. Indirect porcelain veneers have advantages like color stability, wear resistance and biocompatibility with periodontium [6]. Nowadays, ceramic is the main restorative material used in veneer treatments. Just same as natural teeth these veneers reflect and transmit light and show normal illumination [7].

\section{Case Report}

In this case report, we presented a case for 35 years old male patient who had reported to the "Department of Conservative Dentistry and Endodontics at Narsinhbhai Patel dental college and hospital" with chief complaint of brownish staining of upper front teeth. History in detail was taken and extra oral finding were normal. In intraoral examination diagnosis was confirmed that maxillary anterior teeth have fluoride stains. Then vitality test confirmed that all the teeth were vital. And then it was determined to use indirect veneers due to the conservative preparation and patient's desire.

Preoperative clinical photograph were taken first (Fig. 1) and diagnostic casts were obtained with the impression of both arches. Then shade was selected using a color guide (VITA Tooth guide 3D-MASTER, Zahnfabrik H Rauter GmbH and Co KG Spitalgasse, Germany). First of all depth cutter diamond bur was taken and grooves of depth orientation was made on according to it on facial surface of all decided teeth. Approximately $0.3 \mathrm{~mm}$ depth was created on gingival third of teeth and $0.5 \mathrm{~mm}$ on incisal third of teeth. Then the second bur used was round tapered

Kailash A | Volume 2; Issue 3 (2021) | JDHOR-2(3)-036 | Case Report

Citation: Modi N, et al. Veneers - Indirectly Fabricated Dental Porcelain for Aesthetic. J Dental Health Oral Res. 2021;2(3):1-6.

DOI: http://dx.doi.org/10.46889/JDHOR.2021.2305 
diamond bur to remove the remaining extra structure of tooth. The finish line which was created at the gingival crest level was chamfer finish line (Fig. 2). Once the preparation was completed, polyvinyl siloxane was the material of choice for taking putty wash impression. Final shade selection with other all instructions were given to the dental technician along with the putty impression. Then refractory stone models of the prepared teeth were made in dental laboratory followed by fabrication of EMax veneers. Final try in of EMax veneers was done for proper finish margin in the gingival crest level in the clinic. Then the final step of cementation was carried out in the patient's mouth (Fig. 3). After the satisfaction of final try in, the veneers were etched with $5 \%$ hydrofluoric acid for $20 \mathrm{~s}$. Then veneers were cleaned with water and dried. Silane-coupling agent was applied in the interior surface of veneers and waited for 1 minute to dry. Then, the etchant used was $37 \%$ phosphoric acid on all the prepared teeth for approximately 20 seconds and then washed it with water thoroughly, and dried. Then with the help of applicator tips bonding agent was applied and light cured for 20 seconds, For final cementation RelyX; 3M ESPE dual cure resin cement was used. This cement was directly applied on the inner surfaces of veneers and then veneers were applied on the prepared tooth surfaces with mild pressure. Due to complete fit surplus cement came out which was cleaned with the normal probe. Then light was directly applied on veneers for curing. First of all lingual side was polymerized followed by facial side for 1 minute and then other areas of veneers for 30-40 s. To avoid any high point occlusion, contact areas were examined during protrusive movements.

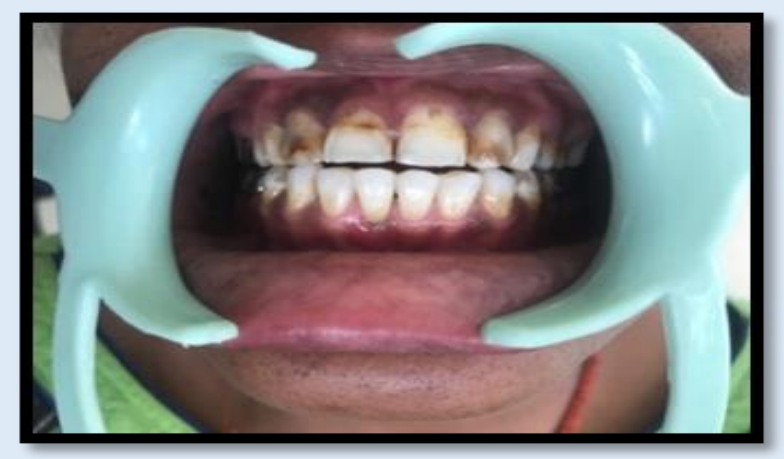

Figure 1: Preoperative photograph.

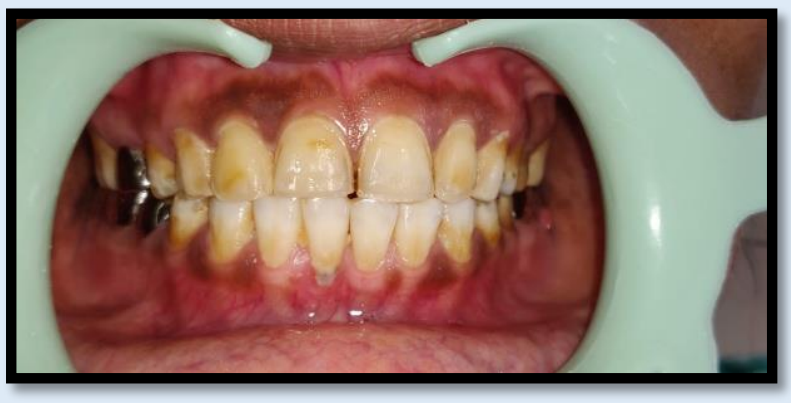

Figure 2: Tooth preparation.

Kailash A | Volume 2; Issue 3 (2021) | JDHOR-2(3)-036 | Case Report

Citation: Modi N, et al. Veneers - Indirectly Fabricated Dental Porcelain for Aesthetic. J Dental Health Oral Res. 2021;2(3):1-6. 


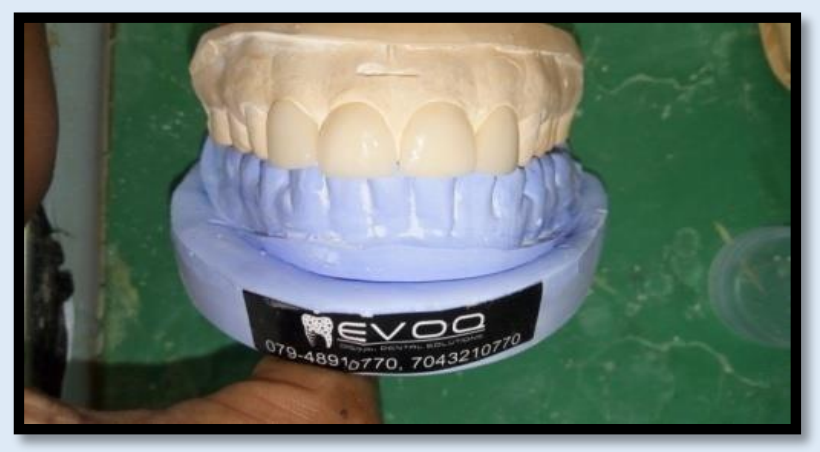

Figure 3: Try in on cast.

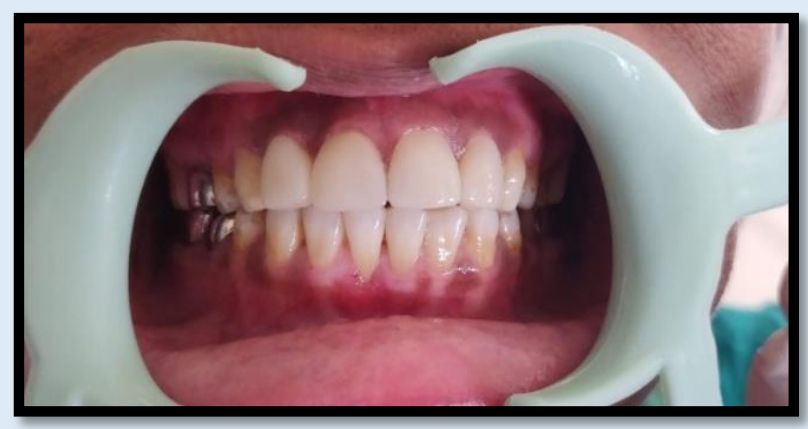

Figure 4: Veneer cementation.

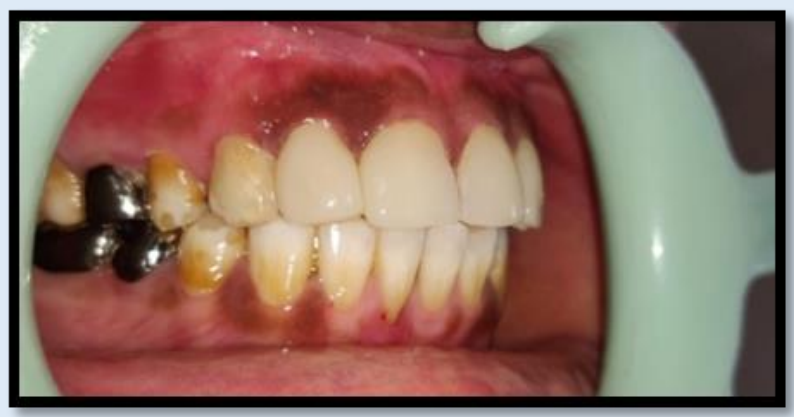

Figure 5: Post-operative.

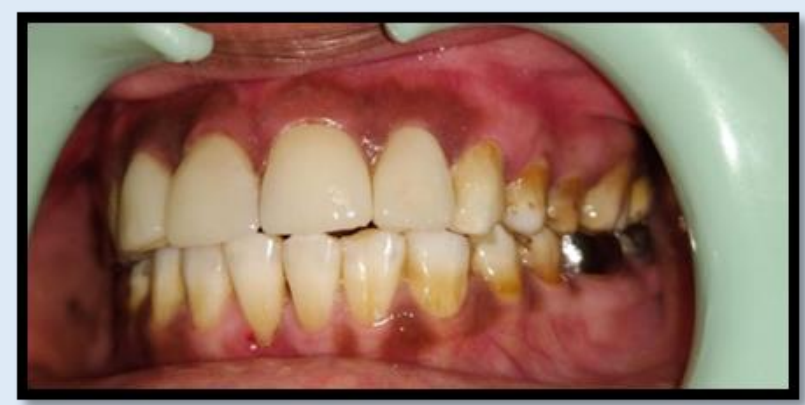

Figure 6: Post-operative.

Kailash A | Volume 2; Issue 3 (2021) | JDHOR-2(3)-036 | Case Report

Citation: Modi N, et al. Veneers - Indirectly Fabricated Dental Porcelain for Aesthetic. J Dental Health Oral Res. 2021;2(3):1-6. 


\section{Discussion}

Prior to decide between direct and indirect veneers several factors such as time, socioeconomical status, oral hygiene of patient have to be considered. Both are very good options for anterior aesthetic problems now a days [5]. Out of all the available treatment option most accepted treatment for correction of anterior teeth with aesthetic is indirect fabricated dental porcelain restorations [5]. Different techniques like total crowns or conservative like indirect veneers are available for anterior rehabilitation. But both total crown and indirect veneer require multiple steps in clinic as well as laboratory [6]. Ceramic laminates can change the complete aesthetic with shape, size, shade of teeth and thickness up to 0.2 to $0.5 \mathrm{~mm}$. Porcelain veneers are accomplished of as long as an tremendously reliable replica of natural teeth with immense colour steadiness [3]. Due to elusive character of indirectly fabricated porcelain veneers cracking is a chief possible post-operative obstacle. The long-standing achievement of porcelain veneers rely on lot of criteria such as proper selection of case, treatment procedure including shade selection, preparation of tooth and type of cement and cementation procedure. Also depends upon patient preservation [3]. Indirect porcelain veneers are not advisable if enamel is not sufficient or any unfavourable anatomy or parafunction of teeth and should also be avoided in patients with poor oral hygiene [7]. Post-operative instructions should be completely advised by dental surgeon to the patient. Patient is advised to take care with tougher food to reduce fractures and dislodgement of veneers and also asked to maintain oral hygiene [8].

\section{Conclusion}

With good enough knowledge of the indirect veneers and proper technique we can give best aesthetic result but should take care due to technique sensitive characteristic of veneers. This treatment is an exceptionally adaptable clinical method that provides advantages of outstanding aesthetic excellence, minimum cutting, biocompatible, patient's happiness and negligible occurrence of dental caries. For all this reason, psychological encouragement with improved confidence is also seen with patients' smile. Based on this case report we can conclude that indirectly fabricated veneers are most satisfactory treatment option for patient as well as dentist for long term success.

\section{Conflict of Interest}

It is stated that there are no conflicts of interest between the proponents and participants in the present work.

Kailash A | Volume 2; Issue 3 (2021) | JDHOR-2(3)-036 | Case Report

Citation: Modi N, et al. Veneers - Indirectly Fabricated Dental Porcelain for Aesthetic. J Dental Health Oral Res. 2021;2(3):1-6. 


\section{References}

1. Soni R, Vivek R. Esthetic rehabilitation by porcelain laminate-a case report. Int J Appl Dent Sci. 2015;1(4):98-100.

2. Mancini M, Mancini M. Ceramic veneers: a step-by-step case report. Global J Oral Sci. 2016;2(2016):20-7.

3. Dahiya S, Ahlawat M, Gandhi A, Khetarpal A, Gill M, Singhal N. Aesthetic correction of discolored fluorosed anteriors with porcelain laminate veneers: Case report. Int J Oral Care Res. 2020;8(1):14.

4. Sowmya K, Dwijendra KS, Pranitha V, Roy KK. Esthetic rehabilitation with direct composite veneering: a report of 2 cases. Case Rep Dentistry. 2017;2017.

5. Korkut B, Yanıkoğlu F, Günday M. Direct composite laminate veneers: three case reports. J Dent Res Dent Clin Dent Pros. 2013;7(2):105.

6. Albuquerque PP, Moreno MB, Nishida AC, Rodrigues E, Kiyohara C, Francci CE. Prefabricated resin veneer: A case report of a simplified restorative technique. J Dent Res Dent Clin Dent Pros. 2018;12(2):140.

7. Sachdeva H, Kumar SK, Sthapak A. Porcelain laminates veneers: case report. J Res Adv Dent. 2015;4(2):1620.

8. Nobrega AS, Signoreli AF, Mazzaro JV, Zavanelli RA, Zavanell AC. Minimally invasive preparations: contact lenses. J Advan Clin Res Insights. 2015;2(4):176-9. 